\title{
Pattern recognition approach to quantify the atomic structure of graphene
}

Kling, Jens; Vestergaard, Jacob Schack; Dahl, Anders Bjorholm; Stenger, Nicolas; Booth, Tim ; Bøggild, Peter; Larsen, Rasmus; Wagner, Jakob Birkedal; Hansen, Thomas Willum

\section{Published in:}

Carbon

Link to article, DOI:

10.1016/j.carbon.2014.03.013

Publication date:

2014

Document Version

Peer reviewed version

Link back to DTU Orbit

Citation (APA):

Kling, J., Vestergaard, J. S., Dahl, A. B., Stenger, N., Booth, T., Bøggild, P., Larsen, R., Wagner, J. B., \& Hansen, T. W. (2014). Pattern recognition approach to quantify the atomic structure of graphene. Carbon, 74 , 363-366. https://doi.org/10.1016/j.carbon.2014.03.013

\section{General rights}

Copyright and moral rights for the publications made accessible in the public portal are retained by the authors and/or other copyright owners and it is a condition of accessing publications that users recognise and abide by the legal requirements associated with these rights.

- Users may download and print one copy of any publication from the public portal for the purpose of private study or research.

- You may not further distribute the material or use it for any profit-making activity or commercial gain

- You may freely distribute the URL identifying the publication in the public portal 


\section{Pattern recognition approach to quantify the atomic structure of graphene}

2 Jens Kling ${ }^{*}, 1,5$, Jacob S. Vestergaard², Anders B. Dahl², Nicolas Stenger ${ }^{3,5}$, Tim J. Booth ${ }^{4}$, Peter

3 Bøggild ${ }^{4,5}$, Rasmus Larsen², Jakob B. Wagner ${ }^{1}$, Thomas W. Hansen ${ }^{1,5}$

$4 \quad{ }^{1}$ Center for Electron Nanoscopy (DTU Cen), Technical University of Denmark, Fysikvej 307, 2800

5 Kgs. Lyngby, Denmark

$6 \quad{ }^{2}$ Department of Applied Mathematics and Computer Science (DTU Compute), Technical University

7 of Denmark, Matematiktorvet 303B, 2800 Kgs. Lyngby, Denmark

$8{ }^{3}$ Department of Photonics Engineering (DTU Fotonik), Technical University of Denmark, Ørsteds

$9 \quad$ Plads 343, 2800 Kgs. Lyngby, Denmark

$10{ }^{4}$ Department of Micro- and Nanotechnology (DTU Nanotech), Technical University of Denmark,

11 Ørsteds Plads 345E, 2800 Kgs. Lyngby, Denmark

$12{ }^{5}$ Center for Nanostructured Graphene (CNG), Technical University of Denmark, Ørsteds Plads

13 345E, 2800 Kgs. Lyngby, Denmark

15 We report a pattern recognition approach to detect the atomic structure on high-resolution

16 transmission electron microscopy images of graphene. The approach provides quantitative 17 information such as carbon-carbon bond lengths and bond length variations on a global and local 18 scale alike.

20 Graphene is considered a key material for future electronic applications with the possibility of very

21 high performance transistors [1], spintronics [2] and ballistic devices even at room temperature [3].

22 The degree to which the actual performance of graphene devices can live up to the theoretical 23 predictions depends critically on the presence of defects, atomic configuration of edges. In essence, 24 any deviations from perfect lattice periodicity can be important, which for instance is manifested in 25 the sensitivity of electronic properties to strain [4].

\footnotetext{
* Corresponding author. E-mail: jenk@cen.dtu.dk (J. Kling), Phone: +4545256487
} 
1 Transmission electron microcopy (TEM) in general and high-resolution TEM (HRTEM) in

2 particular can provide information about the atomic structure and defect landscape of graphene [5].

3 While important parameters like the carbon-carbon (C-C) bond length are possible to determine,

4 this is usually done manually in small areas [6], due to time-consuming work of manually analyzing

5 the HRTEM images. Here we describe a method for fast, automatic structure detection in graphene

6 in a large number of sequentially acquired HRTEM images. The method enables quantitative

7 information such as C-C bond length or bond length variations to be determined from images in a

8 fast and reliable way, and can be used on many images to allow access to this information from a

9 large area.

10 Suspended single-layer graphene synthesized by chemical vapor deposition (CVD) (Graphenea,

11 Spain) or by mechanical exfoliation of graphite [7] and transferred to TEM grids have been

12 investigated using the automatic method. The graphene is imaged using a FEI Titan 80-300

13 Environmental TEM (ETEM) equipped with a monochromator at the electron gun and a spherical

14 aberration (Cs)-corrector for the objective lens. All images are acquired with the microscope operated at $80 \mathrm{kV}$, which is below the knock-on threshold of carbon atoms in pristine graphene [8]. In order to optimize the imaging conditions and thereby the input for the structure detection, the electron beam energy spread was reduced to below $0.3 \mathrm{eV}$ using the monochromator, while the Cs corrector was aligned to minimize the spherical aberration Cs. These conditions result in a resolution better than $0.12 \mathrm{~nm}$, allowing us to resolve the 110 -reflections of graphene and visualize the atomic structure accurately. The images are recorded using a Gatan US1000 CCD camera with 21 an exposure time of $1 \mathrm{~s}$.

22 The structure determination algorithm involves several steps. Utilizing Fourier transformation and 23 local maxima detection, the mean graphene structure over the whole image is detected and used as 24 starting point. The basis is a triangular lattice (triangulation) with a side length of roughly $0.247 \mathrm{~nm}$, connecting three hexagonal centers (nodes) of the graphene structure. Hexagon center positions in the image are recognized as contrast extremes, minima for negative Cs or maxima for positive Cs.

27 Nodes and triangles are removed from the triangulation when the local contrast properties or 
1 geometry deviate significantly from the expectations, and consequently areas like holes or

2 amorphous material can be automatically omitted. In a final step, the node positions are adjusted by

3 grid matching [9]. These steps enable a full reconstruction of the atomic structure of the observed

4 graphene area in most cases. A more detailed description of the algorithm will be published

5 elsewhere.
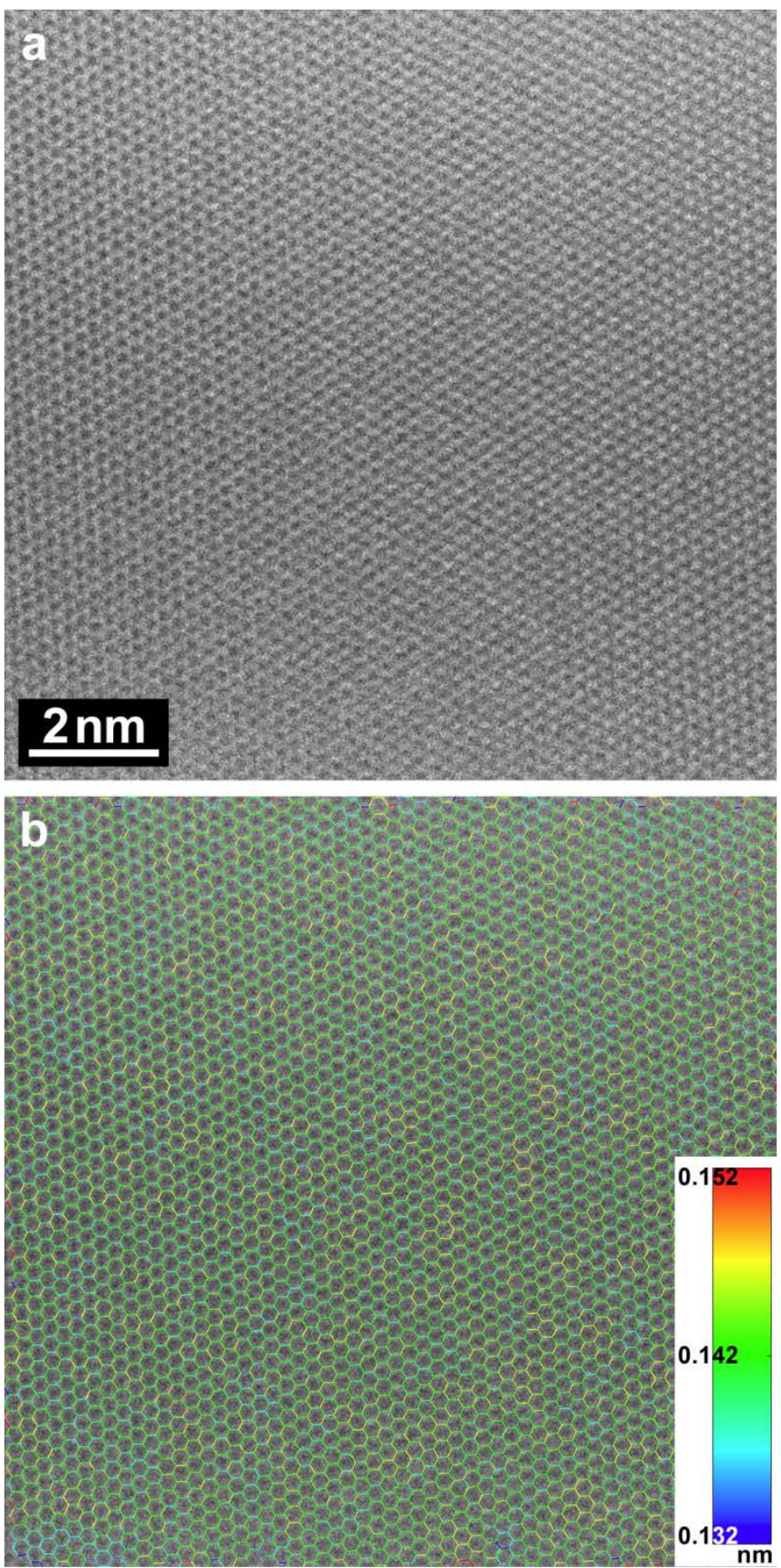

7 Figure 1: Pristine graphene. (a) HRTEM image, (b) image overlaid with the detected and

8 reconstructed graphene structure. The color coding represents the C-C bond lengths. A homogenous

9 distribution of bond lengths is observed. 

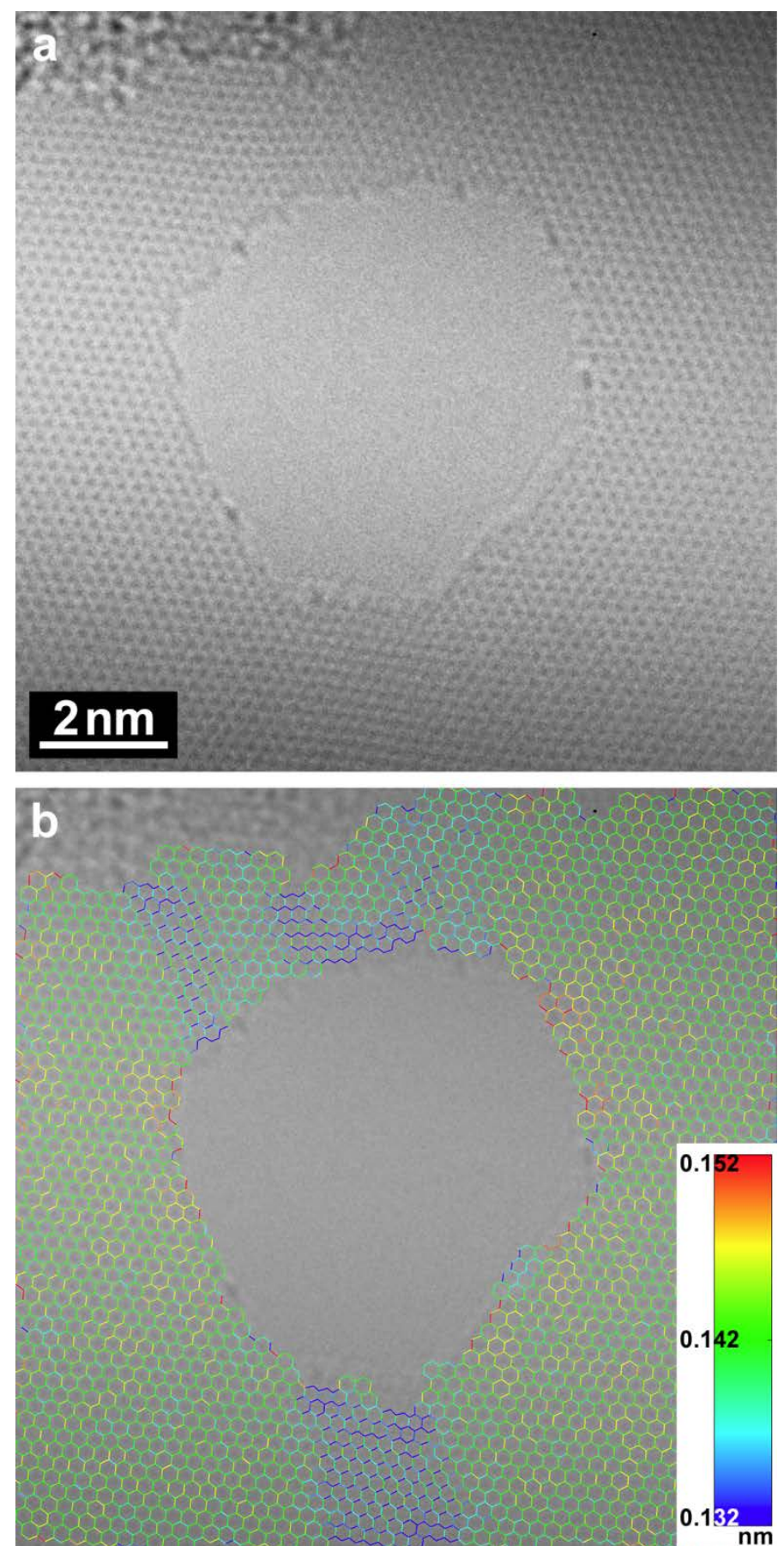

2 Figure 2: Graphene with a hole, formed under the influence of the electron beam. (a) HRTEM

3 image, (b) image overlaid with the detected and reconstructed graphene structure. Color coding is

4 the same as in Fig. 1b. Significantly shorter bond lengths with preferred orientation are observed

5 above and below the hole.

6

7 Figures 1a and 2a show HRTEM images of two different areas of graphene. The hexagonal

8 honeycomb lattice is easily recognized. Using negative Cs imaging, the carbon atoms are bright 9 spots, with the centers of the hexagons appearing dark. In Fig. 1a, a hole in the graphene, formed 
1 under the influence of the electron beam is observed. Due to the inherent small signal-to-noise

$2(\mathrm{~S} / \mathrm{N})$ ratio in the graphene image [5], as well as the continuous beam induced changes of the atomic 3 structure at the edge [10], the termination of the hole cannot be completely resolved. Nevertheless, a 4 predominant zigzag termination is assumed, which is in agreement with previous findings [11]. In

5 Fig. $1 \mathrm{~b}$ and $2 \mathrm{~b}$, the reconstructed graphene structure determined from the algorithm is overlaid, 6 showing the actual hexagonal lattice of the graphene. The color coding represents the C-C bond 7 lengths. As the absolute value retrieved from the images is dependent on the imaging conditions and 8 the calibration, only the relative change within an image is considered. Figure 1b, representing a 9 pristine area, gives the impression of a homogenous distribution of bond lengths. This is reflected in 10 the bond length histogram (Fig. 3 red) which exhibits a normal distribution.

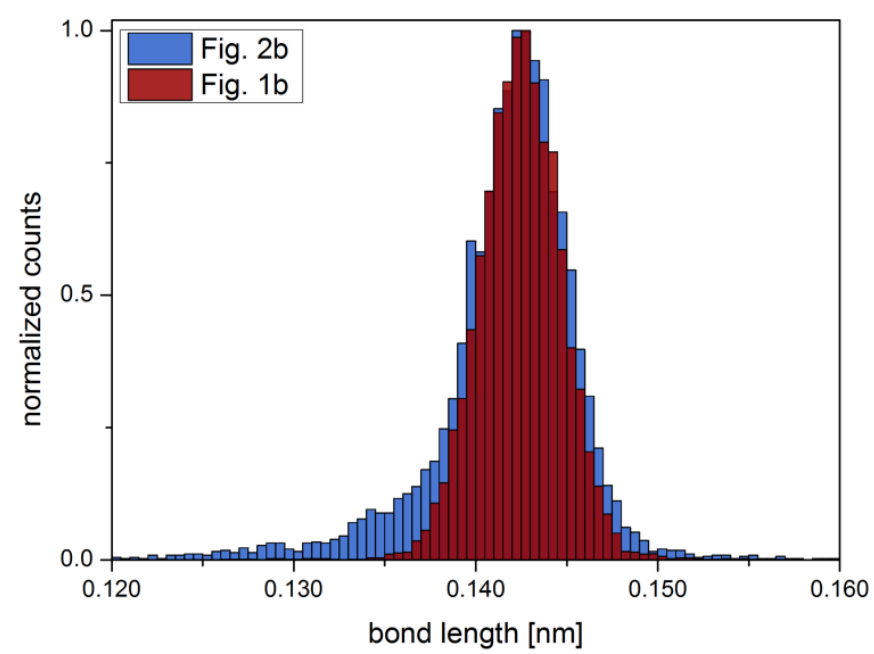

12 Figure 2: Histograms of the detected bond lengths from Fig. 1b (red) and Fig. 2b (blue). The bond lengths are binned by $0.5 \times 10^{-3} \mathrm{~nm}$ steps; the counts are normalized for simpler shape comparison

14 between the two histograms. The red histogram shows a normal distribution. The blue histogram exhibit a tail towards shorter bond length.

17 For the case of a defective structure, as in Fig. 2, the algorithm detects nearly all graphene hexagons. The only exceptions are structures close to the hole and the edge termination itself, which most likely is due to insufficient imaging conditions, as mentioned above. The area of amorphous carbon from the transfer process or synthesis (top left) was disregarded manually, but the area of the 
1 hole is detected by the algorithm and automatically left out in the analysis. A homogenous

2 distribution of bond lengths is observed to the left and right of the hole. For the areas above and

3 below the hole, significantly shorter bond lengths are detected. This is obvious in the histogram as

4 well (Fig. 3 blue), where a tail towards shorter bond length is visible. Suspended graphene is known

5 to form out-of-plane ripples [12], which are expected to have a lateral size of $2-20 \mathrm{~nm}$ and a

6 height of $0.2-1 \mathrm{~nm}$, with an inclination of $\approx 5^{\circ}$ from the horizontal flat sheet. In this case, the

7 projection of the bond length is measured from the image, making the lengths appear shorter. The

8 shortest observed bond lengths above and below the hole have a preferred orientation, almost

9 horizontal in this image. This may be explained by the graphene sheet being slightly folded, as this

10 should lead to a change in z-height as well as elastic deformation and strain mainly in the direction

11 perpendicular to the fold, as we observe in the image. For an almost flat sheet, these bond lengths

12 would represent a strain of about $7-8 \%$; a pure inclination without bond length change would give

13 an angle of about $22^{\circ}$ between the two adjacent carbon atoms and an offset in height of about 0.05

$14 \mathrm{~nm}$. The rearrangement and knock-out of carbon atoms under the electron beam at the edge of the

15 hole [10] can lead to structural deformation as well. A combination of both, a real shorting of the

16 bond length and an artificial shortening due to projection in the image, is the most realistic

17 explanation of our finding. It is important to notice, that even though the shorter bond lengths are

18 represented in the global histogram, the local information, where these bonds actually appear in the structure, is only available in the image itself. The acquired local information in the bond length indicates a possible correlation between measured short bond lengths and folding of graphene. The

21 nature of the bond length shortening will be clarified in a future study.

22 The automated structure detection for quantitative information extraction from high-resolution TEM

23 images is possible for a large amount of images at relatively low time-cost and minimum manual

24 interaction, making it easier and more feasible to follow structural changes in a series of images.

25 Incremental and time dependent structural changes caused by either electron beam induced effects 26 and/or external stimuli like temperature, current etc. can be monitored and quantified in future 27 investigations in greater depths than standard image analysis procedures allow. 
2 The Center for Nanostructured Graphene is sponsored by the Danish National Research Foundation,

3 Project DNRF58. Financial support of the 7th Framework project "GRAFOL" is gratefully

4 acknowledged. The A.P. Møller and Chastine Mc-Kinney Møller Foundation is acknowledged for

5 their contribution toward the establishment of the Center for Electron Nanoscopy in the Technical

6 University of Denmark. Thanks to Graphenea (San Sebastian, Spain) for providing the graphene

7 sample. N. Stenger acknowledges financial support by a Lundbeck Foundation Grant Nbr. R95-

8 A10663.

9 [1] Schwierz F. Graphene Transistors: Status, Prospects, and Problems. Proc IEEE

$10 \quad$ 2013;101:1567-84.

11 [2] Tombros N, Jozsa C, Popinciuc M, Jonkman HT, van Wees BJ. Electronic spin transport and 12 spin precession in single graphene layers at room temperature. Nature 2007;448:571-4.

13 [3] Wang L, Meric I, Huang PY, Gao Q, Gao Y, Tran H, et al. One-dimensional electrical 14 contact to a two-dimensional material. Science 2013;342:614-7.

15 [4] Pereira V, Castro Neto A. Strain Engineering of Graphene’s Electronic Structure. Phys Rev $16 \quad$ Lett 2009;103:046801.

17 [5] Meyer JC, Kisielowski C, Erni R, Rossell MD, Crommie MF, Zettl A. Direct imaging of 18 lattice atoms and topological defects in graphene membranes. Nano Lett 2008;8:3582-6.

19 [6] Warner JH, Margine ER, Mukai M, Robertson AW, Giustino F, Kirkland AI. Dislocation20 driven deformations in graphene. Science (80- ) 2012;337:209-12.

21 [7] Booth TJ, Blake P, Nair RR, Jiang D, Hill EW, Bangert U, et al. Macroscopic graphene membranes and their extraordinary stiffness. Nano Lett 2008;8:2442-6.

23 [8] Zobelli A, Gloter A, Ewels C, Seifert G, Colliex C. Electron knock-on cross section of 24 carbon and boron nitride nanotubes. Phys Rev B 2007;75:245402.

25 [9] Hartelius K, Carstensen JM. Bayesian grid matching. IEEE Trans Pattern Anal Mach Intell 26 2003;25:162-73. 
1 [10] Kotakoski J, Santos-Cottin D, Krasheninnikov A V. Stability of Graphene Edges under Electron Beam: Equilibrium Energetics versus Dynamic Effects. ACS Nano 2012;6:671-6.

3 [11] Girit CO, Meyer JC, Erni R, Rossell MD, Kisielowski C, Yang L, et al. Graphene at the edge: stability and dynamics. Science 2009;323:1705-8.

5 [12] Meyer JC, Geim AK, Katsnelson MI, Novoselov KS, Booth TJ, Roth S. The structure of 6 suspended graphene sheets. Nature 2007;446:60-3. 